Check for updates

Cite this: Nanoscale Adv., 2019, 1, 3957

\title{
Unveiling the interfacial electrochemiluminescence behavior of lead halide perovskite nanocrystals $\uparrow$
}

\author{
Linghang Qu, $+^{a}$ Longhui Lin,,$_{+}^{a}$ Yipeng Huang, ${ }^{a}$ Zhiwei Lai, ${ }^{a}$ Feiming Li, ${ }^{a}$ Shuya Wang, ${ }^{a}$ \\ Fangyuan Lin, ${ }^{a}$ Jianfeng Li, (D) ${ }^{a}$ Yiru Wang ${ }^{a}$ and Xi Chen (DD *abc \\ In this study, a three-phase heterostructure interface including glassy carbon (conducting medium), \\ $\mathrm{CsPbBr}_{3}$ perovskite nanocrystals (PNCs, emitter) and acetonitrile (electrolyte) is constructed for fully \\ investigating the interfacial electrochemiluminescence (ECL) behavior of $\mathrm{CsPbBr}_{3} \mathrm{PNCs}$. We find that \\ these interfaces serve as bridges for efficient electron-hole transfer during the ECL process. As a proof \\ of concept, the increase of the heterostructure interface area will accordingly enhance the $E C L$ intensity \\ of $\mathrm{CsPbBr}_{3} \mathrm{PNCs}$. About seven-fold enhancement of the $\mathrm{ECL}$ intensity could be achieved when the \\ interface area has triple-fold increase, which provides a new perspective to construct more efficient $\mathrm{ECL}$ \\ systems via interface engineering
}

Received 24th July 2019

Accepted 31st August 2019

DOI: $10.1039 / c 9 n a 00456 d$

rsc.li/nanoscale-advances

phase ECL. Unfortunately, deep insights into the corresponding

\section{Introduction}

Lead halide perovskite nanocrystals (LHPNCs) have attracted tremendous attention due to their remarkable optical properties including high brightness, tunable emission wavelength, narrow band emission, and high defect tolerance, which are promising emitters in the field of electrochemiluminescence (ECL). ${ }^{1-5}$ Since the ECL phenomena of $\mathrm{CsPbBr}_{3}$ PNCs in dichloromethane were discovered in 2016, ${ }^{6}$ relevant studies have focused on the ECL behavior of perovskites. Typically, the influence of the sequence of electron and/or hole injection processes on the ECL of $\mathrm{CH}_{3} \mathrm{NH}_{3} \mathrm{PbBr}_{3}$ NCs was investigated. ${ }^{7}$ In addition, the lead-free perovskite, $\mathrm{Cs}_{3} \mathrm{Bi}_{2} \mathrm{Br}_{9}$ QDs was introduced to investigate the ECL activities. ${ }^{8}$ Furthermore, hydrogen peroxide $\left(\mathrm{H}_{2} \mathrm{O}_{2}\right)$ was introduced as a model of reactive oxygen species (ROS) to investigate the ECL of $\mathrm{CsPbBr}_{3}$ PNCs. $^{9}$ In order to achieve better ECL performance, Xue et al. proposed a scraping coating method to acquire high-quality $\mathrm{CsPbBr}_{3} \mathrm{PNC}$ film and introduced anhydrous ethyl acetate as an anti-solvent and co-reactive agent. ${ }^{10}$ Recently, Li et al. simultaneously encapsulated $\mathrm{CsPbBr}_{3}$ QDs and a coreactant into an in situ generated $\mathrm{SiO}_{2}$ matrix to achieve an efficient and stable ECL. ${ }^{11}$ These pioneering studies demonstrate that LHPNCs could serve as a promising candidate for ECL, especially for the organic

\footnotetext{
${ }^{a}$ Department of Chemistry, The MOE Key Laboratory of Spectrochemical Analysis \& Instrumentation, College of Chemistry and Chemical Engineering, Xiamen University, Xiamen 361005, China. E-mail: xichen@xmu.edu.cn

${ }^{b}$ State Key Laboratory of Marine Environmental Science, Xiamen University, Xiamen 361005, China

'Shenzhen Research Institute of Xiamen University, Xiamen 361005, China

$\dagger$ Electronic supplementary information (ESI) available. See DOI: 10.1039/c9na00456d

$\ddagger$ These authors contributed equally.
} ECL process of LHPNCs are still unknown.

As is well-known, the whole ECL process should satisfy three indispensable conditions: $:^{12-15}$ (1) the injection of the electron (or hole) to the emitter and the coreactant; (2) the generation of oxidative species and reductive species; (3) the combination of intermediates to produce the excited state, along with the generation of the ECL signal. In addition, the inherent optical properties of the emitter are the basic insurance of ECL response. In order to reduce the surface energy of the PNCs, ligands are generally introduced to passivate PNCs during their synthesis. ${ }^{16-21}$ However, the existence of flooded long-chain ligands on the PNC surface results in limited conductivity, which greatly hinders the occurrence of the first condition mentioned above. In addition, our earlier study on the $\mathrm{CsPbBr}_{3}$ PNC ECL also shows that the type and content of the surface ligands of $\mathrm{CsPbBr}_{3}$ PNCs do have a significant impact on the ECL intensity. ${ }^{13}$ Furthermore, the increase of the film layer thickness also reduces the interface conductivity, which greatly affects the ECL response. Correspondingly, in order to improve the conductivity of the film layer, previous reports proposed that efficient charge transport can be achieved via crystallized 2D perovskites in a vertically orientated way. ${ }^{22,23} \mathrm{Li}$ et al. added carbon nanotubes into the perovskite film to reduce the interface resistance and promote the charge conduction. ${ }^{24}$ Besides the field of ECL, similar interfacial problems also exist in the construction of photoelectric devices such as perovskite lightemitting diodes (peLEDs). ${ }^{25-31}$ The same obstacle of conductivity limitation prevails in both fields, which may lead to some common rethinking of the PNC film fabrication. Apparently, all these studies reveal that the ECL characteristics of $\mathrm{CsPbBr}_{3}$ PNCs also depend heavily on interfacial structures. However, limited research has been conducted to explore the ECL 
characteristics at the interfaces. Hence, unveiling the interfacial ECL characteristics of $\mathrm{CsPbBr}_{3}$ PNCs is quite important for a better understanding of the ECL process and therefore for constructing more efficient ECL systems.

Herein, in view of the importance of the study of interfacial ECL characteristics, a three-phase interface of electrodeemitter-electrolyte has been established by a partially uncovered $\mathrm{CsPbBr}_{3}$ PNC film on a glassy carbon electrode (GCE) surface (Fig. 1). The constructed three-phase interfaces enable the interfacial electrons (or holes) to be effectively injected into the perovskite layer. As illustrated in Fig. 1, the strong reducing intermediate generated from the coreactant, tripropylamine (TPrA), reacts with the strong oxidant of $\mathrm{CsPbBr}_{3}^{*}$ PNCs, to produce the excited state of $\mathrm{CsPbBr}_{3}$ PNCs and then generate the ECL at the constructed interfaces. Accordingly, the ECL response will be enhanced with the increase of the three-phase interfaces. Moreover, enhanced ECL signal could also be observed on a thicker perovskite film due to the larger area of the exposed three-phase interface since the thickness of the film at the micron level increases with increasing grain boundary, which gives new insights in interface engineering for perovskite films.

\section{Experimental}

\section{Methods}

Chemicals and reagents. Cesium carbonate $\left(\mathrm{Cs}_{2} \mathrm{CO}_{3}\right)$ and alpha,alpha'-dibromo- $p$-xylene (Dbpx) were purchased from Energy Chemical Reagent Co., Ltd (Shanghai, China). Oleylamine (OAm), lead stearate $\left(\mathrm{PbSt}_{2}\right)$ and tripropylamine (TPrA) were obtained from Aladdin (Shanghai, China). 1-Octadecene (ODE) was purchased from Alfa Aesar (China). Acetonitrile was purchased from Shanghai Chemical Reagent Co., Ltd. All reagents were of analytical grade expect OAm and ODE, which were redistilled.

Apparatus and characterization. Ultraviolet absorption spectra were recorded using a Shimadzu UV-2550 and an F-7100 spectrophotometer (Hitachi, Japan) was employed to collect

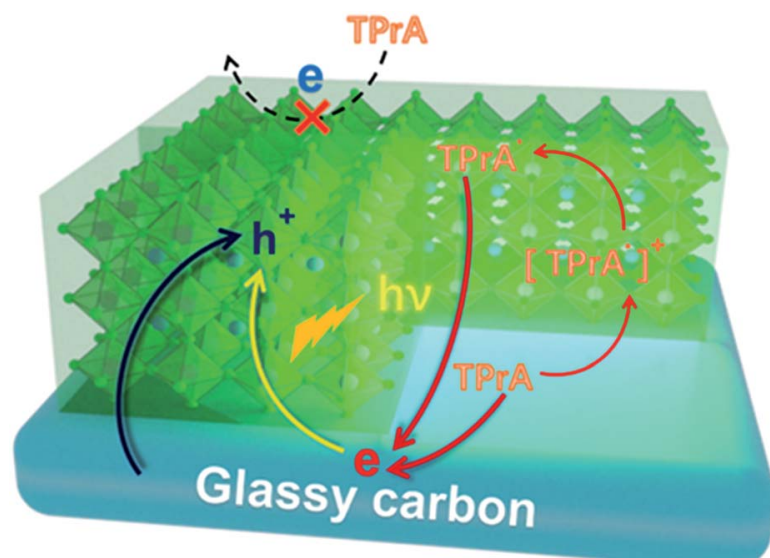

Fig. 1 Schematic of $E C L$ reactions on the three-phase interface consisting of glassy carbon, $\mathrm{CsPbBr}_{3}$ PNCs and acetonitrile (containing $10 \mathrm{mM}$ TPrA). fluorescence emission spectra. The morphologies of samples were observed by scanning electron microscopy (SEM, Hitachi S4800, Japan) and transmission electron microscopy (TEM) with a JEM-1400 microscopy system (JEOL, Japan) at an acceleration voltage of $120 \mathrm{kV}$. AFM images were acquired using a Cypher S atomic force microscope (AFM, Oxford instruments, Britain) in contact mode. X-ray diffraction (XRD) spectra were recorded using a Rigaku Ultima IV instrument (Kuraray, Japan) with a $\mathrm{Cu}$ target $(\lambda=1.54051 \AA)$ operated at $40 \mathrm{kV}$ and $15 \mathrm{~mA}$. The ECL response was recorded with a model MPI-A ECL analyzer from Xi'an Remax Electronic Science \& Technology Co. Ltd (Xi'an, China) combined with a CHI 660C electrochemistry workstation from Shanghai $\mathrm{CH}$ Instruments (Shanghai, China) to perform electrochemical measurements simultaneously. The photomultiplier tube (РМT) was biased at $600 \mathrm{~V}$. A conventional threeelectrode system was introduced in the process, which comprised a modified GCE $(\Phi=3 \mathrm{~mm})$ as the working electrode, $\mathrm{Ag} / \mathrm{AgCl}$ (saturated $\mathrm{KCl}$ solution) as the reference electrode, and a platinum wire as the counter electrode.

Preparation of $\mathbf{C s P b B r}_{3} \mathbf{P N C s} \mathrm{CsPbBr}_{3}$ PNCs were prepared by a simple one-step heating method after optimization according to a previous report. ${ }^{32}$ Specifically, ODE $(5 \mathrm{~mL}), \mathrm{OAm}$ (0.75 mL), $\mathrm{PbSt}_{2}(0.05 \mathrm{mmol}), \mathrm{Dbpx}(0.2 \mathrm{mmol})$ and $\mathrm{Cs}_{2} \mathrm{CO}_{3}$ (0.05 mmol) were sequentially added into a $25 \mathrm{~mL}$ two-neck flask. The mixture was heated from room temperature to 150 ${ }^{\circ} \mathrm{C}$ under intense agitate and then transferred to an ice bath for immediately cooled down. The product was centrifuged at $10000 \mathrm{rpm}$ for $10 \mathrm{~min}$ to discard the supernatant, and then the precipitates were washed with hexane two times by centrifugation. Finally, the precipitates were dried in a vacuum drying oven for subsequent use.

Preparation of $\mathrm{CsPbBr}_{3}$ PNCs|GCE. A GCE was polished with $0.3 \mu \mathrm{m}$ alumina slurry, ultrasonically washed using ultrapure water and then dried in a nitrogen atmosphere. $5 \mu \mathrm{L}$ of $0.1 \mathrm{~g}$ $\mathrm{mL}^{-1}$ colloidal $\mathrm{CsPbBr}_{3}$ PNC slurry dispersed in hexane was dropped on the GCE surface to construct a $\mathrm{CsPbBr}_{3} \mathrm{PNC}$ film for further ECL measurement.

\section{Results and discussion}

The obtained $\mathrm{CsPbBr}_{3}$ PNCs display a distinct excitonic absorption peak around $504 \mathrm{~nm}$ and a sharp photoluminescence (PL) peak at $511 \mathrm{~nm}$ with a full width at halfmaximum (FWHM) of $18 \mathrm{~nm}$, demonstrating a bright green emission under $365 \mathrm{~nm}$ UV light (Fig. 2a). In addition, the $\mathrm{CsPbBr}_{3} \mathrm{PNCs}$ present good monodispersity with an average size of $13 \mathrm{~nm}$ and could be well dispersed in hexane, which is beneficial for the $\mathrm{CsPbBr}_{3}$ PNC film fabrication. In this study, a $\mathrm{CsPBr}_{3} \mathrm{PNC}$ film was fabricated by drop-coating $5.0 \mu \mathrm{L}$ of colloidal $\mathrm{CsPbBr}_{3}$ PNC slurry $\left(0.1 \mathrm{~g} \mathrm{~mL}^{-1}\right)$ on a GCE surface. The film was then left to dry naturally (Fig. 2b) for the subsequent ECL investigation. $\mathrm{CsPbBr}_{3}$ PNCs were dropped on the electrode surface with a high concentration compared with those reported in the literature..$^{6,79}$ In addition, the relatively slow process of solvent evaporation with the appropriate amount of ligands on $\mathrm{CsPbBr}_{3}$ PNCs makes it relatively easier to fabricate a compact film. The scanning electron microscopy (SEM) image 

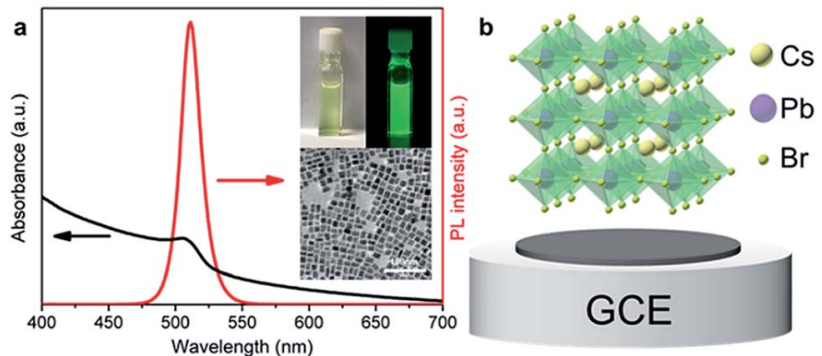

Fig. 2 (a) PL (red line) and UV-vis absorption (black line) spectrum of $\mathrm{CsPbBr}_{3} \mathrm{PNCs}_{\mathrm{S}}$ dispersed in hexane. Inset: the upper one shows $\mathrm{CsPbBr} 3 \mathrm{PNCs}_{3}$ under visible light (lamp off) and UV light (365 nm, lamp on); the bottom one shows the TEM image of $\mathrm{CsPbBr}_{3}$ PNCs. (b) Schematic diagram of $\mathrm{CsPbBr}_{3}$ PNCs|GCE.

shown in Fig. S1a $\uparrow$ illustrates that the $\mathrm{CsPBBr}_{3}$ PNC film on the GCE is quite compact with a micron level thickness. The surface roughness of the $\mathrm{CsPbBr}_{3}$ PNC film was further characterized using atomic force microscopy (AFM). As shown in Fig. S2, $\uparrow$ the AFM image clearly reveals that the surface roughness is around $2 \mathrm{~nm}$. These results confirm the compact and pin-hole free surface of the $\mathrm{CsPBBr}_{3}$ PNC film (Fig. S2a $\dagger$ ), which is quite important for the three-phase interface construction.

In this study, ECL tests were performed in acetonitrile solution containing $10 \mathrm{mM}$ TPrA. In the ECL process, acetonitrile was introduced as the electrolyte on account of its favorable conductivity and the low solubility of $\mathrm{CsPbBr}_{3}$ PNCs. Owing to the presence of the insulating oleylamine (OAm) ligands on the surface of $\mathrm{CsPbBr}_{3}$ PNCs, electrons are severely blocked in the electrolyte, which impedes electron injection into the $\mathrm{CsPbBr}_{3}$ layer and the GCE is covered with $\mathrm{CsPbBr}_{3}$ PNCs in a compact manner. This point could be confirmed well from the undetectable current and ECL signal using the GCE with a compactly covered film of $\mathrm{CsPbBr}_{3}$ layer (Fig. 3b). A control experiment was

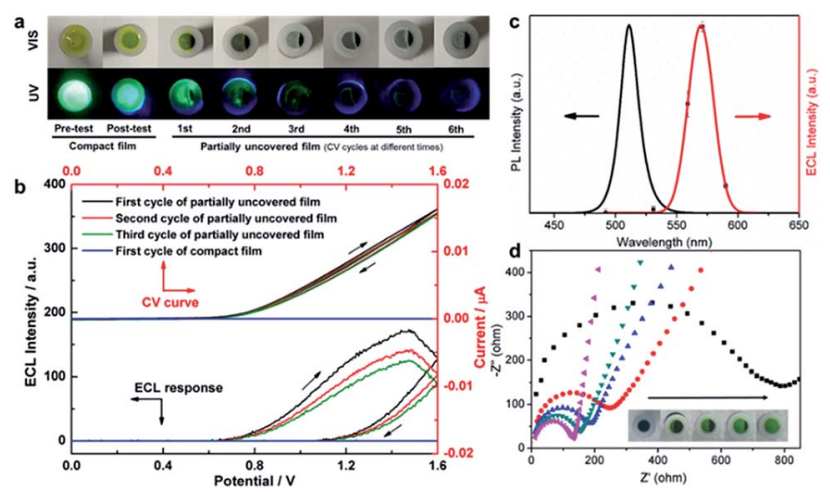

Fig. $3 \mathrm{ECL}$ response of $\mathrm{CsPbBr}_{3}$ PNCs|GCE in acetonitrile containing $10 \mathrm{mM}$ TPrA under continuous cycling scans from $0 \mathrm{~V}$ to $1.6 \mathrm{~V}$. The scan rate was set at $0.05 \mathrm{~V} \mathrm{~s}^{-1}$. (a) Pattern changes of $\mathrm{CsPbBr}_{3}$ PNCs|GCE before and after several CV cycles. Top row images were taken under visible light, and bottom row images were taken under $365 \mathrm{~nm}$ UV light. (b) The ECL emission and cyclic voltammograms from the compact film and partially uncovered film under cycle scans for one to three cycles, respectively. (c) PL (black line, left) and ECL (red line, right) spectra of $\mathrm{CsPbBr}_{3}$ PNCs|GCE. (d) EIS curve of $\mathrm{CsPbBr}_{3}$ PNCs|GCE with different areas of exposed glassy carbon. conducted in the absence of TPrA (Fig. 3b). There is also no ECL response with the compact film. Surprisingly, the electrolytic current and ECL signal obviously increased when the $\mathrm{CsPbBr}_{3}$ film was partially uncovered by gently scraping the compact film to remove the partial-film (Fig. $3 \mathrm{~b}$ ). In addition, compared with the compactly covered film, the electrochemical impedance spectrum (EIS) reveals obvious impedance decrease when a small amount of the $\mathrm{CsPbBr}_{3}$ film was removed to expose the GCE surface (Fig. 3d). This result indicates that the formation of the interface among $\mathrm{CsPbBr}_{3}$ PNCs, glassy carbon and acetonitrile (containing TPrA) opens up the electron or hole transmission channel, and thus triggers the whole reaction. After the ECL measurement, the $\mathrm{CsPbBr}_{3}$ film remains compact (Fig. S1b†), indicating that the electrolyte has failed to infiltrate into the film and the ECL signal merely generates on the interfaces.

As a classical coreactant, the addition of TPrA significantly enhances the anodic ECL signal. In a specific reaction process, with a continuous positive scan from $0 \mathrm{~V}$ to $1.6 \mathrm{~V}$ in the presence of TPrA, $\mathrm{CsPbBr}_{3}$ is transformed to a strong oxidizing intermediate $\left[\mathrm{CsPBBr}_{3}\right]^{+\cdot}$ and TPrA is transformed to a strong reducing intermediate, $\operatorname{TPrA}^{*}$, respectively. $\mathrm{TPrA}^{\circ}$ is the electron donor at the three-phase interface, and the partially uncovered glassy carbon interface acts as an electron transmission bridge, which combines with $\left[\mathrm{CsPbBr}_{3}\right]^{+\cdot}$ to generate an excited state $\mathrm{CsPbBr}_{3}^{*}$ and finally produce the ECL. Without the three-phase interface, electron transmission is blocked, which inhibits ECL generation.

$$
\begin{gathered}
\mathrm{CsPbBr}_{3}-\mathrm{e}^{-} \rightarrow\left[\mathrm{CsPbBr}_{3}\right]^{+\cdot} \\
\mathrm{TPrA}-\mathrm{e}^{-} \rightarrow \operatorname{TPrA}^{\cdot}+\mathrm{H}^{+}
\end{gathered}
$$$$
\left[\mathrm{CsPbr}_{3}\right]^{+\cdot}+\mathrm{TPrA}^{\cdot} \rightarrow \mathrm{CsPbBr}_{3}^{*}+\text { products }
$$$$
\mathrm{CsPbBr}_{3}^{*} \rightarrow \mathrm{CsPbBr}_{3}+h v
$$

The ECL emission peak was found to be $569 \mathrm{~nm}$ with a FWHM of $26 \mathrm{~nm}$, showing a red shift of $58 \mathrm{~nm}$ compared with the PL emission of $\mathrm{CsPbBr}_{3}$ PNCs (Fig. 3c). Such a red shift illustrates that there is a difference between the ECL and PL processes. In general, ECL performance is closely related to the surface state which may lead to a red shift. ${ }^{33}$ On one hand, the impact of ligands cannot be ignored as they reflect the surface properties of $\mathrm{CsPbBr}_{3}$ PNCs and influence the electron transfer process. On the other hand, the secondary growth of $\mathrm{CsPBrr}_{3}$ PNCs on the surface leads to an increase in the size of $\mathrm{CsPbBr}_{3}$ PNCs. It changes the surface state of the $\mathrm{CsPbBr}_{3}$ PNCs and causes the ECL red shift. Since the ECL experiments were carried out in acetonitrile and the potential was repeatedly applied, as shown in Fig. 3a, the color of the $\mathrm{CsPbBr}_{3}$ PNC film gradually changed from green-yellow to white with increase in the cycle number in the cyclic voltammetry (CV) measurements. The corresponding ECL responses also show an attenuated 
tendency (Fig. 3b). Following the verification of the $\mathrm{CsPbBr}$ film, the frequent re-construction of the surface may cause response attenuation, relative to the dissolution and recrystallization processes because of the low solubility of $\mathrm{CsPbBr}_{3}$ in acetonitrile.

In order to verify the presumption that $\mathrm{CsPbBr}_{3}$ PNCs undergo reconstruction on the electrode surface, SEM images of the $\mathrm{CsPbBr}_{3}$ film before and after ECL measurements were observed. As shown in Fig. 4a-c, a smooth surface could be found for the pristine film, while the bulk crystals at the micron level appeared at the boundary after 5 continuous cycles of the potential scan from $0 \mathrm{~V}$ to $1.6 \mathrm{~V}$. The X-ray diffraction (XRD) patterns of the $\mathrm{CsPbBr}_{3}$ films after $\mathrm{CV}$ scans show no new diffraction peaks compared with that of the pristine $\mathrm{CsPbBr}_{3}$ film (Fig. 4d). The typical diffraction peaks at $15.3^{\circ}, 21.7^{\circ}$ and $30.7^{\circ}$ correspond to the (100), (110) and (200) crystal facets of the cubic $\mathrm{CsPbBr}_{3}$ nanocrystals (PDF\#54-752) in these samples, which indicates that the $\mathrm{CV}$ process is not related to the phase transformation of $\mathrm{CsPbBr}_{3}$. Therefore, the reconstruction of $\mathrm{CsPbBr}_{3}$ on the surface is only correlated with the change in crystal size. To further prove this observation, the bromine content in the electrolyte after the CV scan was determined using the reported halide exchange strategy. ${ }^{4}$ In the determination, after the CV scan (5 cycles) the electrolyte was added to the $\mathrm{CsPbI}_{3}$ PNC dispersion (Fig. S4 $\dagger$ ). Eventually, the PL peak exhibited a significant blue shift, indicating that $\mathrm{Br}^{-}$was indeed partially dissolved in the electrolyte during the ECL reaction. For comparison, the electrode covered by the $\mathrm{CsPbBr}_{3}$ film was soaked in acetonitrile for $5 \mathrm{~min}$ without galvanization (the time period is equivalent to the time for CV scan). When pure acetonitrile was directly added to the dispersion solution, a small red shift of the PL peak of $\mathrm{CsPbI}_{3}$ PNCs could be observed since acetonitrile acts as an anti-solvent to agglom-

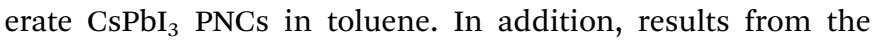

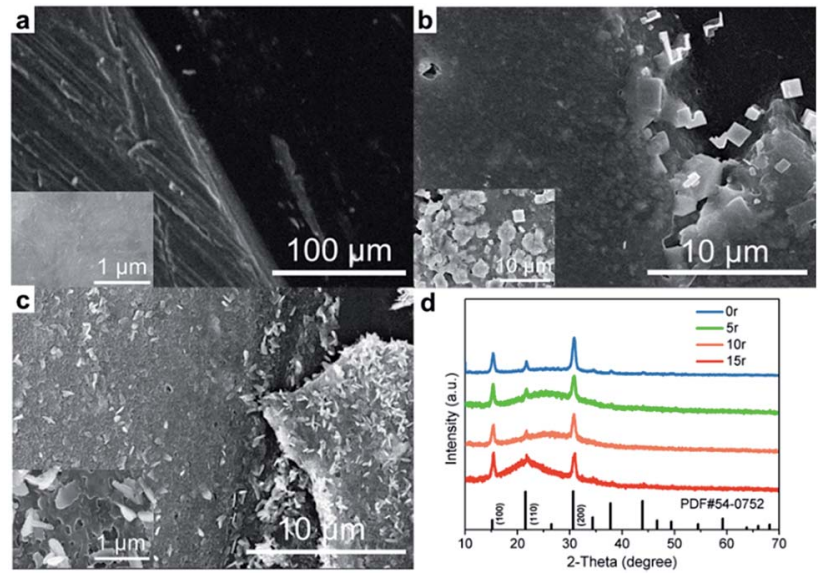

Fig. $4 \mathrm{SEM}$ image of $\mathrm{CsPbBr}_{3}$ PNCs|GCE with half area exposure of the electrode surface under cyclic voltammetry scanning steps from 0 to $1.6 \mathrm{~V}$ for (a) 0 ; (b) 5; (c) 10 cycles. Insets show surface conditions around the boundary between the exposed glassy carbon interface and $\mathrm{CsPbBr}_{3}$ PNC film. (d) XRD patterns of $\mathrm{CsPbBr}_{3}$ PNCs subjected to $\mathrm{CV}$ cycle steps from 0 to $1.6 \mathrm{~V}$ for $0,5,10,15$ times, respectively. All scan rates are set at $0.05 \mathrm{~V} \mathrm{~s}^{-1}$.
Energy Dispersive Spectrum (EDS) experiment also confirm the loss of bromine (Fig. S5 $\dagger$ ). With the increase of cycle scan number, the percentage of $\mathrm{Br}$ atoms at the three-phase interface decreases continuously. The absence of $\mathrm{Br}^{-}$verifies the regrowth of the layer in the reaction. In addition, the small amount of $\mathrm{CsPbI}_{3} \mathrm{PNCs}_{\mathrm{s}}$ on the electrode surface compared with a relatively large amount of electrolyte also leads to partial ligand dissolution. ${ }^{34}$ Therefore, obvious aggregation results in secondary growth and micron scale crystals. With continuous CV scanning, the $\mathrm{CsPbBr}_{3}$ PNC film undergoes fragmentation and pore formation gradually. After 10 cycles, plenty of lamellae appearing on the film surface indicate the collapse of the perovskite structure and the continuous decrease of the corresponding ECL response. From the observation of the film structure during the ECL process, it is found that $\mathrm{CsPbBr}_{3} \mathrm{PNC}$ films are always subject to the trend of constant remodeling and disintegration. Therefore, it is necessary to adopt bulk perovskites or a thicker film to achieve relatively enduring and stable ECL signals due to the stability of the perovskite in the electrolyte and the resistance to the potential erosion.

In order to investigate the effect of the three-phase interface exposure area on the ECL response, a three-phase interface was fabricated semi-quantitatively by properly uncovering the perovskite film from the same electrode surface (Fig. 5). Different removing patterns for the three-phase interface construction are described in Fig. S6. $\dagger$ The corresponding electrochemical active area $(A)$ could be estimated following the previous report (Table $\mathrm{S} 1 \dagger) .{ }^{35}$ In the process of continuous removal, we found that the ECL response on removing a quarter of the $\mathrm{CsPbBr}_{3}$ PNC film (pattern I) is close to that of half removal (pattern II, Fig. 5a). However, EIS curves, as shown in Fig. S7, $\uparrow$ reveal that the impedance decreased obviously with the exposure area increase of the GCE. The electrode conductivity increase means that there are more electrons taking part in the ECL reaction. In fact, whether it is the quarter-removed or the half-removed film (pattern I/pattern II), the effective exposure area of the three-phase interface is almost the same due to the
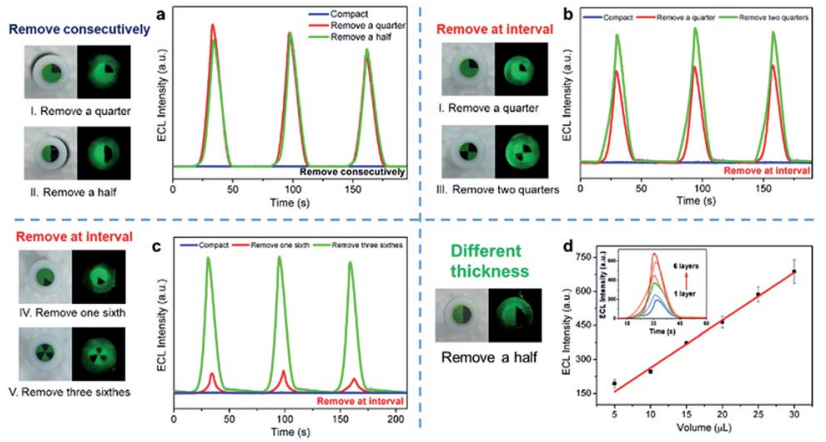

Fig. 5 Three-phase interface construction and corresponding ECL response. The interfaces were constructed with two film removal models: (a) consecutive removal; (b and c) removal at intervals. (d) Different thicknesses by multi-layer drop-coating from 1 layer to 6 layers with removing a half of the film. All experiments under continuous cycling scans from 0 to $1.6 \mathrm{~V}$ in acetonitrile, the scan rate was $0.05 \mathrm{Vs}^{-1}$. 
same length of the two radii and the thickness of the layer $(5 \mu \mathrm{L}$ $\mathrm{CsPbBr}_{3} \mathrm{PNCs}_{\mathrm{N}}$ coated). Considering the ECL response in the continuous removal mode (Fig. 5a), it is manifested that the number of electrons involved in the reaction on the three-phase interface is limited. The extra electrons do not participate in the ECL reaction, but cause increase in the conductivity. On the other hand, in the mode of removing at intervals (Fig. 5b and c), ECL response is enhanced for the two quarters-removed film (pattern III) compared with the quarter-removed film (pattern I) as shown in Fig. 5b. Moreover, the exposed area of the two quarters-removed film on the GCE surface (pattern III) is the same as the half-removed film (pattern II) while the effective area of the three-phase interface is different. That is, the former interface involves a length of about four radii and a thickness of one layer. It is thus clear that the ECL response of the perovskite film has no significant relationship with the exposed area of the GCE surface, but is closely related to the effective three-phase interface. Similarly, as shown in Fig. 5c, comparing the threesixth-removed film (pattern $\mathrm{V}$ ) by interval removal with the one sixth-removed film (pattern IV) the strength increases by nearly sevenfold, which further reveals the importance of the three-phase interface exposure. In addition, as shown in Fig. S8, $\uparrow$ the ECL intensity is in nearly direct proportion to the number of three-phase interfaces which reiterates the interface model for the ECL of $\mathrm{CsPbBr}_{3}$ PNCs.

In addition, the ECL responses show a linear increase trend as the boundary thickness of the $\mathrm{CsPbBr}_{3} \mathrm{PNC}$ film prepared by multi-layer drop-coating along with the half-removed film as shown in Fig. 5d. The sectional view of the electrode with different layers is recorded in the SEM images (Fig. S9†). Combined with the interfacial behavior of the ECL reaction as aforementioned, we speculate that the glassy carbon (electrode) injects holes into the $\mathrm{CsPbBr} \mathrm{PHC}_{3}$ film and produces sufficient $\mathrm{TPrA}^{\circ}$ radicals (coreactant in the electrolyte), thereby the thicker $\mathrm{CsPbBr}_{3} \quad \mathrm{PNC}$ film (emitters) produces positive radicals $\left[\mathrm{CsPbBr}_{3}\right]^{+\cdot}$ to accept more electrons from TPrA ${ }^{\bullet}$ radicals on the three-phase interface, which ultimately increases the ECL response.

\section{Conclusions}

Previous studies on the ECL of perovskites have always focused on electrochemical processes rather than the electrode interfaces. In this study, we revealed the interfacial ECL characteristics of perovskite nanocrystals in acetonitrile, and properly established the relationship between the construction of a three-phase interface and ECL response. We verified that with the increase of three-phase interfaces, that is, the more interfaces meeting with the essential conditions of ECL, stronger ECL signals could be obtained. This work provides a new idea to explore the ECL characteristics of perovskite, presenting a unique angle for interface engineering construction of perovskite film.

\section{Conflicts of interest}

There are no conflicts to declare.

\section{Acknowledgements}

This research work is financially supported by the National Natural Science Foundation of China (21876141, 21675133), the Foundation for Innovative Research Groups of the National Natural Science Foundation of China (Grant No. 21521004), and the Shenzhen Science and Technology Project (No. JCYJ20180306172823786).

\section{Notes and references}

1 T. Xuan, J. Huang, H. Liu, S. Lou, L. Cao, W. Gan, R.-S. Liu and J. Wang, Chem. Mater., 2019, 31, 1042-1047.

2 Y. Li, X. Wang, W. Xue, W. Wang, W. Zhu and L. Zhao, Nano Res., 2019, 12, 785-789.

3 M. V. Kovalenko, L. Protesescu and M. I. Bodnarchuk, Science, 2017, 358, 745-750.

4 Q. A. Akkerman, V. D'Innocenzo, S. Accornero, A. Scarpellini, A. Petrozza, M. Prato and L. Manna, J. Am. Chem. Soc., 2015, 137, 10276-10281.

5 C. Zhou, Y. Tian, O. Khabou, M. Worku, Y. Zhou, J. Hurley, H. Lin and B. Ma, ACS Appl. Mater. Interfaces, 2017, 9, 40446-40451.

6 Y. Huang, M. Fang, G. Zou, B. Zhang and H. Wang, Nanoscale, 2016, 8, 18734-18739.

7 X. Tan, B. Zhang and G. Zou, J. Am. Chem. Soc., 2017, 139, 8772-8776.

8 Y. Cao, Z. Zhang, L. Li, J. R. Zhang and J. J. Zhu, Anal. Chem., 2019, 91, 8607-8614.

9 Y. Huang, X. Long, D. Shen, G. Zou, B. Zhang and H. Wang, Inorg. Chem., 2017, 56, 10135-10138.

10 J. Xue, Z. Zhang, F. Zheng, Q. Xu, J. Xu, G. Zou, L. Li and J. J. Zhu, Anal. Chem., 2017, 89, 8212-8216.

11 L. Li, Z. Zhang, Y. Chen, Q. Xu, J. R. Zhang, Z. Chen, Y. Chen and J. J. Zhu, Adv. Funct. Mater., 2019, 1902533, DOI: 10.1002/adfm.201902533.

12 Q. Zhai, J. Li and E. Wang, ChemElectroChem, 2017, 4, 16391650.

13 Z. Cai, F. Li, W. Xu, S. Xia, J. Zeng, S. He and X. Chen, Nano Res., 2018, 11, 1447-1455.

14 X. Chen, Y. Liu and Q. Ma, J. Mater. Chem. C, 2018, 6, 942959.

15 L. Yang, Y. Li, Y. Zhang, D. Fan, X. Pang, Q. Wei and B. Du, ACS Appl. Mater. Interfaces, 2017, 9, 35260-35267.

16 J. Pan, Y. Shang, J. Yin, M. De Bastiani, W. Peng, I. Dursun, L. Sinatra, A. M. El-Zohry, M. N. Hedhili, A. H. Emwas, O. F. Mohammed, Z. Ning and O. M. Bakr, J. Am. Chem. Soc., 2018, 140, 562-565.

17 B. Luo, Y. C. Pu, S. A. Lindley, Y. Yang, L. Lu, Y. Li, X. Li and J. Z. Zhang, Angew. Chem., Int. Ed. Engl., 2016, 55, 8864-8868.

18 D. N. Dirin, S. Dreyfuss, M. I. Bodnarchuk, G. Nedelcu, P. Papagiorgis, G. Itskos and M. V. Kovalenko, J. Am. Chem. Soc., 2014, 136, 6550-6553.

19 C. Zhang, B. Wang, Q. Wan, L. Kong, W. Zheng, Z. Li and L. Li, Nanoscale, 2019, 11, 2602-2607.

20 J. H. Park, A. Y. Lee, J. C. Yu, Y. S. Nam, Y. Choi, J. Park and M. H. Song, ACS Appl. Mater. Interfaces, 2019, 11, 8428-8435. 
21 L. Wu, Q. Zhong, D. Yang, M. Chen, H. Hu, Q. Pan, H. Liu, M. Cao, Y. Xu, B. Sun and Q. Zhang, Langmuir, 2017, 33, 12689-12696.

22 J. Zhang, L. Zhang, X. Li, X. Zhu, J. Yu and K. Fan, ACS Sustainable Chem. Eng., 2019, 7, 3487-3495.

23 A. Z. Chen, M. Shiu, X. Deng, M. Mahmoud, D. Zhang, B. J. Foley, S.-H. Lee, G. Giri and J. J. Choi, Chem. Mater., 2019, 31, 1336-1343.

24 X. Li, D. Yu, J. Chen, Y. Wang, F. Cao, Y. Wei, Y. Wu, L. Wang, Y. Zhu, Z. Sun, J. Ji, Y. Shen, H. Sun and H. Zeng, ACS Nano, 2017, 11, 2015-2023.

25 M. Yuan, L. N. Quan, R. Comin, G. Walters, R. Sabatini, O. Voznyy, S. Hoogland, Y. Zhao, E. M. Beauregard, P. Kanjanaboos, Z. Lu, D. H. Kim and E. H. Sargent, Nat. Nanotechnol., 2016, 11, 872-877.

26 Z. K. Tan, R. S. Moghaddam, M. L. Lai, P. Docampo, R. Higler, F. Deschler, M. Price, A. Sadhanala, L. M. Pazos, D. Credgington, F. Hanusch, T. Bein, H. J. Snaith and R. H. Friend, Nat. Nanotechnol., 2014, 9, 687-692.
27 G. Li, Z. K. Tan, D. Di, M. L. Lai, L. Jiang, J. H. Lim, R. H. Friend and N. C. Greenham, Nano Lett., 2015, 15, 2640-2644.

28 J. Wang, N. Wang, Y. Jin, J. Si, Z. K. Tan, H. Du, L. Cheng, X. Dai, S. Bai, H. He, Z. Ye, M. L. Lai, R. H. Friend and W. Huang, Adv. Mater., 2015, 27, 2311-2316.

29 R. Long, J. Liu and O. V. Prezhdo, J. Am. Chem. Soc., 2016, 138, 3884-3890.

30 M. Yang, Y. Zeng, Z. Li, D. H. Kim, C. S. Jiang, J. van de Lagemaat and K. Zhu, Phys. Chem. Chem. Phys., 2017, 19, 5043-5050.

31 B. Li, J. Tian, L. Guo, C. Fei, T. Shen, X. Qu and G. Cao, ACS Appl. Mater. Interfaces, 2016, 8, 4684-4690.

32 F. Lin, F. Li, Z. Lai, Z. Cai, Y. Wang, O. S. Wolfbeis and X. Chen, ACS Appl. Mater. Interfaces, 2018, 10, 23335-23343. 33 Z. Ding, B. M. Quinn, S. K. Haram, L. E. Pell, B. A. Korgel and A. J. Bard, Science, 2002, 296, 1293-1297.

34 X. Li, D. Yu, F. Cao, Y. Gu, Y. Wei, Y. Wu, J. Song and H. Zeng, Adv. Funct. Mater., 2016, 26, 5903-5912.

35 P. Podesva, I. Gablech and P. Neuzil, Anal. Chem., 2018, 90, 1161-1167. 